\title{
Complexity and the Big Bang
}

\author{
Hermann Nicolai \\ Max-Planck-Institut für Gravitationsphysik \\ Albert-Einstein-Institut \\ Mühlenberg 1, D-14476 Potsdam, Germany \\ Email: nicolai@aei.mpg.de
}

\begin{abstract}
After a brief review of current scenarios for the resolution and/or avoidance of the Big Bang, an alternative hypothesis is put forward implying an infinite increase in complexity towards the initial singularity. This may result in an effective non-calculability which would present an obstruction to actually reaching the beginning of time. This proposal is motivated by the appearance of certain infinite-dimensional duality symmetries of indefinite Kac-Moody type in attempts to unify gravity with the fundamental matter interactions, and deeply rooted in properties of Einstein's theory.
\end{abstract}


1. Introduction. The beginning of the Universe is shrouded in mystery. Observations indicate that it came into being with a Big Bang, where the spacetime geometry and physical quantities characterizing matter (density, pressure, temperature, etc.) appear to become singular, see e.g. $[1,2]$ for an introduction. It seems generally agreed that the resolution of these singularities will require the reconciliation of general relativity and quantum theory into a consistent theory of quantum gravity. Addressing this challenge should pave the way for a proper understanding of what precisely 'happened' at the cosmic instant $\tau=0$, and answer the question whether the Big Bang represents a true beginning, or whether the history of our universe extends into a possibly infinite past.

In this Note I wish to outline an approach to the problem of the initial singularity that is motivated by the magic link between Einstein's theory and its (supersymmetric) generalizations on the one hand, and certain infinite-dimensional duality symmetries, and the hyperbolic Kac-Moody algebra $\mathfrak{e}_{10}$, in particular, on the other. The main observation is that properties of these algebras may introduce a mathematically well defined element of non-calculability which may forever shield the initial singularity from resolution.

2. Brief survey of previous ansätze. Existing approaches to quantum gravity propose specific ways to deal with the initial singularity, thereby also offering potential answers to the questions posed above:

Creation 'from nothing'. The idea that the universe was created by quantum tunneling from nothing, and without an initial singularity, was put forward already some time ago $[3,4]^{1}$ and motivates the celebrated Hartle-Hawking ansatz for the wave function of the universe [8]. According to the latter, Lorentzian space-time can be explained as emerging from a Euclidean instanton. The switch from Lorentzian to Euclidean signature enables one to 'round off' the past before the Big Bang into a cap, such that the beginning of time can no longer be located, and the singularity simply 'disappears'. However, the precise meaning of the term 'nothing' here raises subtle ontological issues that reach beyond physics, see e.g. [9]: for instance, is this a creation out of literally nothing, or is it a process to be embedded in a larger entity (a megaverse)?

Quantum bounce. Canonical approaches to quantum gravity posit that singularity resolution can be achieved by replacing the classical time evolution of the spatial geometry by the quantum mechanical evolution of a wave function(al)

\footnotetext{
${ }^{1}$ An even earlier variant of this idea is B. DeWitt's suggestion that the wave function of the universe might have vanishing support on singular 3-geometries [5]; see also [6, 7] for more recent results in this direction.
} 
smearing the 3 -geometries over the singular classical trajectories $[10,11,12]$ (for possible realizations of singularity avoidance in the framework of covariant loop quantum gravity, see [13]). The bang would be replaced by a quantum bounce, and preceded by a collapsing universe, and would therefore not represent a beginning of time. The ancestor universe itself must either have originated from an infinite past, or be part of a sequence of repeated bounces. Cosmic bounces are also central to the ekpyrotic universe [14].

Avoiding the singular point. A fundamental theory may admit an operation allowing for the cosmic scale factor $a$ to 'jump over' the singular point, for instance if one can analytically continue its dynamics into the complex $a$-plane. Pre-Big Bang string cosmology relies on the purported UV completeness of string theory to argue that such an operation can be safely implemented, e.g. by means of a $T$ duality transformation linking different 'phases' of space-time [15]. Likewise, but with very different reasoning, Penrose's conformal cyclic cosmology postulates a 'reset' $a=\infty \rightarrow a^{\prime} \equiv a^{-1}=0$ at the end of each 'æon' [16], a process whose dynamical origin remains unclear, however.

Eternal inflation. Many variants of inflationary cosmology [17] are based on the hypothesis that baby universes can arise by quantum mechanical bubble nucleation, a process that is assumed to take place continually. In this picture, a megaverse, of which our universe is merely an infinitesimal part, would simply 'exist' with neither beginning nor end, possibly also allowing for other kinds of universes, as suggested by the string landscape [18]. However, it has been shown that there then always exist geodesically incomplete past directed curves [19]. This excludes the possibility that our universe can be embedded in a megaverse space-time geometry that is completely free of singularities (but see [20]). For the resolution of multiple past and future singularities linked to the appearance and disappearance of baby universes this scenario has to appeal to other ideas.

A common feature of these proposals is that they often rely on the assumption that there is a basic 'simplicity' about the universe and its beginning, thus extrapolating Einstein's cosmological principle to quantum cosmology. This is usually done by restricting the infinitely many degrees of freedom to a finite number of variables (such as the cosmic scale factor and a number of spatially homogeneous fields), or alternatively by means of purely heuristic manipulations of an ill-defined gravitational path integral or Wheeler-DeWitt operator. Contrary to these often stated views, I here want to argue that there is no such 'simplicity' about the beginning of the universe, in the sense that the underlying fundamental theory could exhibit an unbounded complexity towards the 
initial singularity that may turn out to be an inherent and ineluctable feature of quantum gravity.

3. Reformulating gravitational dynamics near the singularity. Before getting to the main point (in section 5) we first need some preparation. We start from the space-time metric (setting the shift to zero)

$$
\mathrm{d} s^{2}=-\left(N \mathrm{~d} x^{0}\right)^{2}+g_{m n} \mathrm{~d} x^{m} \mathrm{~d} x^{n} \quad(m, n, \ldots=1, \ldots, d)
$$

where $d$ is the number of spatial dimensions. Denoting the momenta canonically conjugate to the spatial metric components $g_{m n}$ by $\Pi^{m n}$, the Hamiltonian constraint reads (see e.g. [10])

$$
\mathcal{H}=G_{m n \mid p q}(\mathrm{~g}) \Pi^{m n} \Pi^{p q}-\sqrt{\mathrm{g}} R^{(d)}(\mathrm{g})+\mathcal{H}_{\text {matter }} \approx 0
$$

Here $G_{m n \mid p q}(\mathrm{~g})$ is the DeWitt metric, which is famously indefinite, and the matter part of the Hamiltonian $\mathcal{H}_{\text {matter }}$ need not be specified at this point. For the mathematical analysis of the dynamics near the singularity à la BelinskiKhalatnikov-Lifshitz (BKL) [21] one singles out the logarithmic scale factors $\beta^{a}$, parametrizing the off-diagonal metric components in Iwasawa form [22]

$$
\mathrm{g}_{m n}=\sum_{a=1}^{d} e^{-2 \beta^{a}} \mathcal{N}^{a}{ }_{m} \mathcal{N}^{a}{ }_{n}
$$

where the matrices $\mathcal{N}^{a}{ }_{m}$ are upper triangular, with $\mathcal{N}^{a}{ }_{m}=1$ for $a=m$. It is convenient to choose a gauge for the lapse function $N$ such that, with proper time $\tau$, the time coordinate $t \sim-\log \tau$ becomes 'Zeno-like' (i.e. stretching the finite time interval in $0<\tau<\tau_{0}$ to infinity) and the singularity is located in the infinite future $(t=\infty)$. A detailed analysis now shows that with these variables the Hamiltonian constraint (2), at a given spatial point, and with different matter couplings, can be rewritten as [22]

$$
\mathcal{H}\left(\beta^{a}, \pi_{a}, \ldots\right)=G^{a b} \pi_{a} \pi_{b}+\sum_{A} c_{A}(\ldots) e^{-2 w_{A}(\beta)}
$$

Here, $\pi_{a}$ are the canonical momenta conjugate to the scale factors $\beta^{a}$, and $G_{a b}$ is the Lorentzian metric obtained by restricting the DeWitt metric to diagonal metric degrees of freedom. The dots in (4) stand for all other canonical degrees of freedom (off-diagonal metric components, spatial gradients, matter, etc.).

The exponential terms in (4) involve linear forms $w_{A}(\beta) \equiv w_{A a} \beta^{a}$, where the vectors $w_{A}$ characterize the various contributions to the Hamiltonian, and the prefactors $c_{A}$ are (complicated) functions of the canonical variables other than 
$\beta^{a}$ and $\pi_{a}$, see [22] for details. The walls normal to the vectors $w_{A}$ partition the $\beta$-space into chambers. In the near singularity (BKL) limit the Hamiltonian dynamics simplifies radically, being dominated by a number of leading wall forms $\left\{w_{i} \mid i=1, \ldots, r\right\}$, and is reduced to that of a relativistic billard ball bouncing off the walls of a fundamental chamber in $\beta$-space, see also [23] for an equivalent description. The occurrence (or not) of chaotic metric oscillations depends on the 'shape' of this chamber, where the 'shape' itself depends on the matter content and the number of space-time dimensions: chaotic oscillations happen whenever the billiard wedge is located within the light-cone in $\beta$-space [24]. This description thus unifies previous analyses based on case-by-case studies [25]. ${ }^{2}$

\section{Group theoretical transmutation of the gravitational Hamiltonian.}

The key fact is now that there exists a detailed, though still very incomplete, correspondence of the (matter coupled) gravitational dynamics with a purely group theoretical construct [26]. To exhibit it, one identifies the space of scale factors $\beta^{a}$ with the Cartan subalgebra (CSA) of a certain indefinite Kac-Moody algebra $\mathfrak{g}$; the metric $G_{a b}$ from (4) then becomes the Cartan-Killing metric on the CSA. Crucially, the indefiniteness of the DeWitt metric implies that the Cartan-Killing metric is also indefinite. Identifying the simple roots of $\mathfrak{g}$ with the dominant wall forms $w_{i}$, the Cartan matrix defined by [27]

$$
A_{i j} \equiv G^{a b} w_{i a} w_{j b} \quad(1 \leq i, j \leq r)
$$

is likewise indefinite (for simplicity we take $\mathfrak{g}$ to be simply laced). The dynamics take place on the infinite-dimensional coset space $\mathcal{G} / K(\mathcal{G})$, where $\mathcal{G}$ is the (noncompact) 'group' obtained by formally exponentiating $\mathfrak{g}$, and $K(\mathcal{G})$ its 'maximal compact subgroup'. Adopting an Iwasawa gauge analogous to (3), it is governed by the Hamiltonian

$$
\mathcal{H}_{K M}\left(\beta^{a}, \pi_{a}, \ldots\right)=G^{a b} \pi_{a} \pi_{b}+\sum_{\alpha>0} \sum_{s=1}^{\operatorname{mult}(\alpha)} \Pi_{\alpha, s}^{2}(\ldots) e^{-2 \alpha(\beta)}
$$

where the sum on the r.h.s. ranges over all positive roots $\alpha$ of $\mathfrak{g}$ and their multiplicities (with $\alpha(\beta) \equiv \alpha_{a} \beta^{a}$ ), and $\Pi_{\alpha, s}$ parametrize the non-CSA degrees of freedom. Restricting the dynamics to the leading walls and to the simple roots, respectively, one finds exact agreement between (4) and (6) [22]. More generally, (6) implies null geodesic motion on $\mathcal{G} / K(\mathcal{G})$. The correspondence between the two models can then be shown to extend to first order spatial

\footnotetext{
${ }^{2}$ Because the BKL analysis relies on causal decoupling of spatial points, it applies only to space-like (cosmological) singularities, but not to time-like or null singularities.
} 
gradients for the gravitational Hamiltonian, and to roots $\alpha$ of low height for the coset Hamiltonian (6) [26].

As is well known, the Hamiltonian constraint must be imposed at each spatial point. By contrast, there is no 'space' in (6)! Moreover, instead of finitely many contributions $c_{A}$ to the potential as in (4), the sum on the r.h.s. of (6) has an infinite number of contributions. The conjecture put forward in [26] then amounts to saying that the spatial field theory degrees of freedom now 'reside' in the infinite-dimensional Lie algebra $\mathfrak{g}$. This picture of the evolution as a onedimensional motion is in accord with the fact that cosmological evolution can be described as geodesic motion of a point particle in the moduli space of spatial geometries $[28,29]$ - except that this moduli space is now replaced by the coset space $\mathcal{G} / K(\mathcal{G})$. To be sure, numerous conceptual and mathematical questions remain. Most important, these concern the precise nature of the mechanism by which space and space-time ${ }^{3}$, as well as concomitant features such as general covariance and gauge symmetry, are supposed to emerge from a purely group theoretical construct, and the key role that quantization and the incorporation of fermions are expected to play in it (but see e.g. [30, 6, 31]).

In the remainder I will focus on the most important case, maximal supergravity in eleven space-time dimensions ('M theory') [32, 33], for which $\mathfrak{g}=\mathfrak{e}_{10}$. This is the maximally extended (= maximal rank) hyperbolic Kac-Moody algebra, with 'group' $\mathcal{G}=\mathrm{E}_{10}$, which for myriad reasons is a prime candidate symmetry for a Planck scale unified theory of quantum gravity (but see [34]).

5. Confronting $\mathrm{E}_{10}$ symmetry. The Lie algebra $\mathfrak{e}_{10}$ is recursively defined in terms of generators and relations (Chevalley-Serre presentation) [35]

$$
\begin{gathered}
{\left[h_{i}, e_{j}\right]=A_{i j} e_{j}, \quad\left[h_{i}, f_{j}\right]=-A_{i j} f_{j}, \quad\left[e_{i}, f_{j}\right]=\delta_{i j} h_{j}} \\
\operatorname{ad}\left(e_{i}\right)^{1-A_{i j}}\left(e_{j}\right)=0, \quad \operatorname{ad}\left(f_{i}\right)^{1-A_{i j}}\left(f_{j}\right)=0
\end{gathered}
$$

with ten elementary $\mathfrak{s l}_{2}$ building blocks $\left\{e_{i}, f_{i}, h_{i} \mid i, j=1, \ldots, 10\right\}$, that is, one for each node of the Dynkin diagram (see also [36] for a pedestrian introduction). Here the $\mathrm{E}_{10}$ Cartan matrix $A_{i j}$ follows from (5) (with $r=10$ ), and thus has a truly (super-)gravitational origin [27]. These 'starting rules' are fairly simple to write down, but for indefinite Cartan matrix $A_{i j}$ they hide an unbounded complexity that evolves out of their repeated application (recall that, by contrast, positive definite Cartan matrices lead to finite-dimensional simple Lie algebras, as the iterated commutators terminate after finitely many steps [35]). For in-

\footnotetext{
${ }^{3}$ Since we are working in a Hamiltonian framework, 'time' would have to emerge from the timeless Wheeler-DeWitt equation, in analogy with more standard canonical approaches [10].
} 
definite Cartan matrix there is thus no 'closed form' of this Lie algebra, nor even a list or enumeration of its basis elements. To visualize the complications it is perhaps simplest to think of it as a Lie algebra analog of a Mandelbrot set, but (for all we know) without the self-similar features. The indefiniteness of $A_{i j}$ entails the existence of infinitely many roots, and an exponential increase in the multiplicities of timelike imaginary roots with their length [35]. This feature is reminiscent of the exponential increase in the number of massive states in string theory, but here the growth is much more erratic: unlike for string theory, there is no partition function nor any other known number theoretic device to describe that growth.

To better understand the Lie algebra $\mathfrak{e}_{10}$ one can expand it as a graded direct sum of vector spaces [37]

$$
\mathfrak{e}_{10}=\bigoplus_{\ell=-\infty}^{\infty} \mathfrak{e}_{10}^{(\ell)}
$$

where the level $\ell$ refers to a decomposition in terms of representations of some (preferably finite-dimensional) regular subalgebra $\mathfrak{e}_{10}^{(0)} \subset \mathfrak{e}_{10}$. However, any recursive analysis is prohibitive: the exponential growth of the dimensions of $\mathfrak{e}_{10}^{(\ell)}$ with increasing $\ell$ is clearly evident in the tables of [38]. No matter where one cuts off the expansion (8), this mathematical structure never reaches a stationary state as $\ell \rightarrow \infty$, but keeps getting more complicated!

Exploring the physics of the $\mathrm{E}_{10} / \mathrm{K}\left(\mathrm{E}_{10}\right)$ model one hits upon the very same difficulties that obstruct the mathematical analysis (and more). The nascent complexity of this system is already apparent in the occurrence of chaotic oscillations in the original BKL analysis [21]. These difficulties multiply as one probes degrees of freedom beyond the CSA. Nevertheless, with only the Iwasawa gauge to rely on, exploiting local $\mathrm{K}\left(\mathrm{E}_{10}\right)$ symmetry to restrict the degrees of freedom to non-negative levels in (8), one can show that the different maximal supergravities with their duality symmetries all emerge at low levels, depending on how one 'slices' $\mathfrak{e}_{10}$ in (8) [39, 40, 41]. Still, no investigation has gone beyond $|\ell| \leq 4$ so far, despite many efforts. The correct physical interpretation of the higher level modes remains an enigma.

It is common lore that non-diagonal degrees of freedom 'freeze' in the BKL limit [22]. Furthermore, the wave function can be shown to generically vanish at the singularity in this approximation [6]. Nevertheless, within the full $\mathrm{E}_{10} / \mathrm{K}\left(\mathrm{E}_{10}\right)$ coset manifold this simplification fails (although the evanescence of the wave function at the singularity may still persist). Namely, inspection of the geodesic deviation equation shows that the sectional curvatures decrease 
without limit for imaginary roots $\alpha$, as $\alpha^{2} \rightarrow-\infty$ [42], implying that geodesics on $\mathrm{E}_{10} / \mathrm{K}\left(\mathrm{E}_{10}\right)$ become infinitely unstable as $t \rightarrow \infty$. It is unknown whether quantization and inclusion of fermions (perhaps in bosonized form) can mitigate these instabilities, but they surely affect the 'wave function of the universe'. Irrespective of how close to the singularity and at which depth in $\mathfrak{e}_{10}$ one specifies initial conditions, there always remains an uncontrollably infinite deficit: tracing the path of the cosmic wave packet out of the singularity would require a descent down a bottomless 'devil's staircase'.

6. Complexity and information. Although the preceding discussion provides ample evidence for the complexity of $\mathfrak{e}_{10}$, this concept, though intuitively clear, should be properly defined and quantified. The relevant notion here is algorithmic complexity, which can be (roughly) measured via the minimal time to perform a computation [43] (similarly, in quantum circuits 'complexity' can be defined via the minimal number of quantum gates required to reach a given final state from a given initial one [44]). If we apply this idea to $\mathfrak{e}_{10}$, the 'complexity' of a higher level state associated to an imaginary root $\alpha$ increases without bound as $\alpha^{2} \rightarrow-\infty$ (like the sectional curvature). To capture the intrinsic complexity contained in the individual imaginary root spaces would require a vastly generalized notion of automorphicity, which is not known.

Because the time-reversed picture of the Big Bang is the black hole singularity, the present considerations could also provide a very different perspective on the black hole information paradox. The possible relevance of the BKL analysis in this context was recently highlighted in [45], where the preservation of information is linked to the vanishing of the wave function at the singularity (see above). The new aspect of the present work is that for the full $\mathfrak{e}_{10}$ algebra, the available phase space at the singularity becomes infinitely larger than the BKL phase space of diagonal metrics. Unbounded growth of complexity may furthermore create a basic asymmetry between moving the cosmic wave packet into or moving it out of the singularity, a distinction that has been repeatedly emphasized by R. Penrose, see e.g. [46].

7. Physics? Observational confirmation of any proposal towards resolving the initial singularity is notoriously difficult. An obvious idea is to search for hidden signatures in the CMB, for instance by identifying some peculiar pattern in the cosmic fluctuation spectrum. However, we have only one CMB map, not an ensemble of maps, and this makes it difficult to ascertain whether a perceived special feature is not simply a statistical fluke. Indeed it appears unlikely (to this author) that one will be able to unambiguously pin down the right theory 
of quantum gravity simply 'by looking at the sky'.

Yet, there may be different and unexpected ways to validate the present scheme. A surprising result is that the maximal compact subgroup $K\left(E_{10}\right)$ admits unfaithful finite-dimensional fermionic representations [47, 48, 49, 50]. Remarkably, one of these can be matched with the fermion content of the standard model, with three generations of quarks and leptons (including right-handed neutrinos) [51]; to match the fermionic quantum numbers, $\mathrm{K}\left(\mathrm{E}_{10}\right)$ is absolutely essential $[52,51]$. This is therefore a scheme that can potentially explain the spin- $\frac{1}{2}$ content of the standard model as is, with no extra room for new fundamental spin- $\frac{1}{2}$ degrees of freedom (such as those predicted by low energy supersymmetry), and where supersymmetry is 'superseded' by $\mathrm{E}_{10}$ and $\mathrm{K}\left(\mathrm{E}_{10}\right)$ symmetry. In addition, this scheme predicts eight supermassive spin- $\frac{3}{2}$ fermions (gravitinos) participating in standard model interactions. These may explain the ultrahigh energy cosmic ray events observed over many years [53], and the origin and growth of primordial black holes [54]. Indirectly, $\mathrm{E}_{10}$ and $\mathrm{K}\left(\mathrm{E}_{10}\right)$ could thus help in addressing two major open problems of modern astrophysics.

8. Resumé. The basic claim put forward in this Note can be summarized very simply: even if we were able eventually to formulate a consistent (UV complete) unified theory of quantum gravity and validate its low energy manifestations against observation, the emergent complexity of this theory may prevent us from getting to the bottom of what it predicts. The beginning of time could thus remain beyond the reach of our attempts to understand it.

Acknowledgments: I am most grateful to T. Damour, M. Henneaux, A. Kleinschmidt and K.A. Meissner for collaboration and inspiration over many years, and to C. Kiefer and J.L. Lehners for helpful comments. I would also like to thank the referees for clarifying comments. This work has been supported by the European Research Council (ERC) under the European Union's Horizon 2020 research and innovation programme (grant agreement No 740209).

\section{References}

[1] E.W. Kolb and M.S. Turner, The Early Universe, Addison-Wesley (1990)

[2] V. Mukhanov, Physical Foundations of Cosmology, Cambridge Univ. Press (2005)

[3] R. Brout, F. Englert and E. Gunzig, Annals Phys. 115 (1978) 78

[4] A. Vilenkin, Phys. Lett. B117 (1982) 25 
[5] B.S. DeWitt, Phys.Rev. 160 (1967) 1195; Phys. Rev. 162 (1967) 1239

[6] A. Kleinschmidt, M. Köhn and H. Nicolai, Phys. Rev. D80 (2009) 061701

[7] C. Kiefer, N. Kwidzinski and D. Piontek, Eur. Phys. J. C 79 (2019) 686

[8] J.B. Hartle and S.W. Hawking, Phys. Rev. D28 (1983) 2960

[9] P.C.L. Tang, The ontological status of the cosmological singularity, Springer Verlag (1989)

[10] C. Kiefer, Quantum gravity, Clarendon Press, 2004

[11] M. Bojowald, Phys. Rev. Lett. 86 (2001) 5227

[12] A. Ashtekar and T. Pawlowski, Phys. Rev. Lett. 96 (2006) 141301

[13] C. Rovelli and F. Vidotto, Covariant Loop Quantum Gravity, Cambridge Univ. Press (2014)

[14] J. Khoury, B. Ovrut, P. Steinhardt and N. Turok, Phys. Rev. D64 (2001) 123522

[15] M. Gasperini and G. Veneziano, Astropart. Phys. 1 (1993) 317

[16] R. Penrose, in Proceedings of EPAC 2006, Edinburgh, Scotland

[17] J. Martin, C. Ringeval and V. Vennin, Phys. Dark Univ. 5-6 (2014) 75

[18] A.N. Schellekens, Rev. Mod. Phys. 85 (2013) 1491; Int. J. Mod. Phys. A30 (2015) 1530016

[19] A. Borde, A.H. Guth and A. Vilenkin, Phys. Rev. Lett. 90 (2003) 151301

[20] G.F.R. Ellis and R. Maartens, Class. Quant. Grav. 21 (2004) 223

[21] V. A. Belinsky, I. M. Khalatnikov and E. M. Lifshitz, Adv. Phys. 19 (1970) 525

[22] T. Damour, M. Henneaux and H. Nicolai, Class. Quant. Grav. 20 (2003) R145

[23] C. W. Misner, Phys. Rev. 186 (1969) 1319

[24] T. Damour, M. Henneaux, B. Julia and H. Nicolai, Phys. Lett. B509 (2001) 323

[25] J. Demaret, J.L.Hanquin, M. Henneaux and P. Spindel, Phys. Lett. B175 (1986) 129.

[26] T. Damour, M. Henneaux and H. Nicolai, Phys. Rev. Lett. 89 (2002) 221601 
[27] T. Damour and M. Henneaux, Phys. Rev. Lett. 86 (2001) 4749

[28] J. Greensite, Class. Quant. Grav. 13 (1996) 1339

[29] P.K. Townsend and M. Wohlfarth, Class. Quant. Grav. 21 (2004) 5375

[30] J. Brown, O.J. Ganor and C. Helfgott, JHEP 0408 (2004) 063

[31] T. Damour and P. Spindel, Phys. Rev. D95 (2017) 126011

[32] E. Cremmer, B. Julia and J. Scherk, Phys. Lett. B76 (1978) 409

[33] B. Julia, in: Lectures in Applied Mathematics, Vol. 21 (1985), AMS-SIAM, p. 335

[34] P.C. West, Class. Quant. Grav. 18 (2001) 4443

[35] V. G. Kac, Infinite dimensional Lie algebras, 3rd edition, Cambridge University Press (Cambridge, 1990)

[36] R.W. Gebert and H. Nicolai, $\mathrm{E}_{10}$ for beginners, in Strings and Symmetries, Springer Lecture Notes in Physics, eds. G. Aktas et al. (1994)

[37] A.J. Feingold and I.B. Frenkel, Math. Ann. 263 (1983) 87

[38] H. Nicolai and T. Fischbacher, in Kac-Moody Lie algebras and related topics, eds. N. Stanumoorthy and K.C. Misra, Contemporary Mathematics 343, American Mathematical Society (2004)

[39] A. Kleinschmidt and H. Nicolai, Int. J. Mod. Phys. D15 (2006) 1619

[40] F. Riccioni and P.C. West, JHEP 0707 (2007) 063

[41] E. Bergshoeff, I. DeBaetselier and T. Nutma, JHEP 0709 (2007) 047

[42] T. Damour and H. Nicolai, Class. Quant. Grav. 22 (2005) 2849

[43] I. Wegener, Complexity Theory, Springer-Verlag (2005)

[44] M. Nielsen and J. Chuang, Quantum Computation and Quantum Information, Cambride Univ. Press (2010)

[45] M.J. Perry, No future in black holes, arXiv:2106.03715

[46] R. Penrose, Why current string theory cannot resolve the gravitational singularity issue, talk at Strings 2021, Sao Paulo

[47] T. Damour, A. Kleinschmidt and H. Nicolai, Phys. Lett. B634 (2006) 319

[48] S. de Buyl, M. Henneaux and L. Paulot, JHEP 02 (2006) 056

[49] A. Kleinschmidt, H. Nicolai and A. Viganò, in Partition functions and automorphic forms, eds. V.A. Gritsenko and V.P. Spiridonov, Springer Verlag (2020) 
[50] A. Kleinschmidt, R. Köhl, R. Lautenbacher and H. Nicolai, arXiv: 2102.00870 [math.RT]

[51] K.A. Meissner and H. Nicolai, Phys.Rev. D 91 (2015) 065029; Phys. Rev. Lett. 121 (2018) 091601

[52] A. Kleinschmidt and H. Nicolai, Phys. Lett. B747 (2015) 251

[53] K.A. Meissner and H. Nicolai, JCAP 09 (2019) 041

[54] K.A. Meissner and H. Nicolai, Phys. Rev. D102 (2020) 103008; Phys.Lett. B819 (2021) 136468 